\title{
Analysis of renal function in a Health Examination Population in Chengdu (2012-2018)
}

\section{Rui-Cen Li}

Sichuan University West China Hospital

\section{Ting Bao}

Sichuan University West China Hospital

\section{Han-Wei Yang}

Sichuan University West China Hospital

\section{Wang-Dong Xu}

southwest medical university, luzhou, sichuan, China

Huai-Rong Tang ( $\nabla$ tanghuairong16@163.com )

Sichuan University West China Hospital

\section{Research article}

Keywords: Renal function; Health examination; Epidemiology

Posted Date: May 28th, 2020

DOI: https://doi.org/10.21203/rs.3.rs-30822/v1

License: (c) (i) This work is licensed under a Creative Commons Attribution 4.0 International License. Read Full License 


\section{Abstract}

Background Dysfunction of kidney correlates with multiple renal diseases, for instance, chronic kidney disease. However, monitoring the changes of indexes related to renal function may help to early find the potential patients with renal diseases.

Methods A cohort of 14,716 participants (8,148 males and 6,568 females) was recruited from the Health Examination Center at West China Hospital, Sichuan University, and was followed up since 2012 to 2018. Four indexes related to renal function, including creatinine (Cr), uric acid (UA), cystatin C (CysC) and urea nitrogen (UN) were selected to monitor the kidney function.

Results Levels of CysC, Cr were reduced, and expression of UA was increased in all the participants since 2012 to 2018 overall. In addition, levels of CysC, UA, Cr and UN were higher in males than those in females, and expression of the four indexes was up-regulated in the elderly population compared with those in the younger population since 2012 to 2018 overall.

Conclusions The findings suggested that some preventive action may be adopted early and more attention can focus on the health-examination population.

\section{Introduction}

Kidney disease is an vital cause of morbidity and mortality around the global. One tenth of the world's population suffer from kidney disease, which may be more serious in low-income countries [1]. Numerous factors may contribute to this disorder, including infection, diabetes, obesity and autoimmune diseases. Most patients with kidney injury will develop into chronic kidney disease (CKD) and even lead to renal failure if they are not diagnosed and treated promptly [2]. Recently, with the increasing incidence of CKD worldwide, it has become a serious public health problem [3]. In 2015, about 30 minllion adults in the United States suffered from CKD [4]. The prevalence of CKD in chinese adults is more than $10 \%$ [5]. The global burden of disease study in 2015 showed that CKD was the 17th leading cause of annual mortality worldwide and one of the fastest growing causes of death. From 2005 to 2015, the total mortality rate of CKD increased by $31.7 \%$ [6]. Moreover, the cost of treatment for kidney diseases and related complications is a heavy financial burden on health services around the world. Annual survey revealed that CKD cost about $\$ 35,000$ to $\$ 100,000$ per person per year $[7,8]$. In developing countries, due to infection, hazardous work and insufficient education, CKD poses a greater economic burden [6].

CKD is a chronic disorder characterized by structural or functional abnormalities of kidney, a course of more than 3 months, and presence of markers of kidney damage or decreased glomerular filtration rate (GFR) [9]. The disease is accompanied by a large number of adverse complications, such as cardiovascular disease, progression of nephropathy, acute kidney injury, cognitive decline, skeletal diseases, and increased fracture [1]. These complications collectively increase risk of disability and death $[5,10]$. Early identification and treatment of CKD can help prevent or reduce the risk for CKD-related complications [6]. Indeed, a decline in renal function may lead to the accumulation of toxins and have 
harmful effects on various metabolic pathways, including the progression of inflammation and kidney damage [11]. Thus, it is necessary to measure and monitor renal function of the population. Regular physical examination is a vital means for early detection of CKD and renal dysfunction. In clinical practice, serum creatinine ( $\mathrm{Cr}$ ), uric acid (UA) and cystatin $\mathrm{C}(\mathrm{CySC})$ are widely used to evaluate renal dysfunction [12]. Since $\mathrm{Cr}$ concentration is susceptible to age, gender, race, and diet, which may lead to overestimated glomerular filtration rate (GFR), CysC contains potential advantages as an indicator of renal function as compared to $\mathrm{Cr}$. Serum urea nitrogen (UN) is also of great significance in early diagnosis of kidney diseases $[13,14]$. In present study, we recruited 14,716 subjects from the Health Examination Center at West China Hospital, Sichuan University, and analyzed the annual renal function indicators from 2012 to 2018, including UA, CysC, Cr and UN, to reveal distribution characteristics of renal function level in the population.

\section{Materials And Methods}

\section{Study subjects}

A total of 14,716 participants in this follow-up cohort were recruited from the Health Examination Center at West China Hospital, Sichuan University, consisting of 8,148 males and 6,568 females. All populations took an annual health examination at this hospital from 2012 to 2018. Participants signed informed consent forms before enrollment. Relevant clinical indicators were detected through peripheral blood collection. The present study was approved by Ethics of Research Committee of West China Hospital, Sichuan University.

\section{Laboratory index measurement and data collection}

Demographic characteristics and laboratory data were obtained from annual health examination data and questionnaires. After fasting for 8 to 10 hours, $5 \mathrm{ml}$ venous blood was collected and placed in vacuum tubes containing ethylenediamine tetraacetic acid. Serum was separated by centrifugation and stored for testing. CysC, UA, Cr and UN were detected by immunoturbidimetry, enzymatic colorimetry, picric acid colorimetry and urease method, respectively. Using the results of 2012 as baseline data, the same indicators were detected from 2013 to 2018.

\section{Statistical analysis}

Data analysis was conducted using SAS9.4 software (SAS Institute Inc. Cary, NC, 2013). Quantitative data conforming to normality were described by mean \pm standard deviation (SD). The maximum and minimum values were also exhibited in our study. Classified data was showed by absolute number/percentage. Comparation of quantitative values of each year and the baseline year in 2012 was done by Paired-samples $t$ test. $P<0.05$ indicated statistical significance.

\section{Results}




\section{Characteristics of participants and four renal function indicators}

The characteristics of participants were shown in Table 1. A total of 14,716 individuals were enrolled in the current study, including 8,148 males (55.37\%) and 6,568 females (44.63\%). The average age of health examination populations was $45.12 \pm 13.13$ years old. The mean value of body mass index (BMI) was $23.36 \pm 3.14 \mathrm{~kg} / \mathrm{m}^{2}$. Regarding to systolic blood pressure (SBP) and diastolic blood pressure (DBP), the mean levels were $113.30 \pm 15.34 \mathrm{mmHg}$ and $73.52 \pm 9.68 \mathrm{mmHg}$, respectively.

In 2012, the average level of CysC was $0.91 \pm 0.20 \mathrm{mg} / \mathrm{L}$ in overall population, which decreased to $0.82 \pm 0.18 \mathrm{mg} / \mathrm{L}$ in 2018. Based on the data of 2012, levels of CysC from 2013 to 2018 were lower than that in 2012, respectively (Table 2). However, increased UA concentrations in years 2013 to 2018 were observed compared with that in 2012 (Supplementary Table 1). In addition to Cr level, up to 2018, it was declined to $71.95 \pm 18.38 \mathrm{umol} / \mathrm{L}$ and the levels from 2013 to 2018 were significantly lower than that in 2012 (Supplementary Table 2). UN expressions were higher in years 2013 to 2016 than that in 2012. On the contrary, decreased levels in 2017 and 2018 were observed when compared with that of $2012(t=-7.44$, $\mathrm{P}<0.0001 ; \mathrm{t}=-9.83, \mathrm{P}<0.0001$ ) (Supplementary Table 3).

\section{Distribution of indicators for renal function in different gender}

A total of 8,148 males and 6,568 females were recruited in the present study. In 2012, the mean level of CysC was $0.97 \pm 0.20 \mathrm{mg} / \mathrm{L}$ in males and $0.84 \pm 0.17 \mathrm{mg} / \mathrm{L}$ in females. The values of this indicator in years 2013 to 2018 were significantly lower than that in 2012 both in males and females (Table 3). Regarding to UA, the mean level in each year from 2013 to 2018 was higher than that of 2012, either in males or females. There were significant differences for the concentrations of this indicator between years 20132017 and 2012 both in different gender groups. Notably, the average levels of UA were higher in males when compared with those in females from 2012 to 2018 (Supplementary Table 4). Until 2018, Cr expression was reduced in different gender groups. In general, the index was lower in women than that in men every year (Supplementary Table 5). Furthermore, from 2013 to 2016, elevated average level of UN in males was observed in every year compared with that in 2012. Conversely, in 2017 and 2018, it was declined as compared to that in 2012 (Supplementary Table 6).

\section{Distribution of indicators for renal function in different age groups}

We divided all participants into 7 groups by age (group 1: 20 to 29 years old; group 2: 30 to 39 years old; group 3: 40 to 49 years old; group 4: 50 to 59 years old; group 5: 60 to 69 years old; group 6: 70 to 79 years old; group 7: over 80 years old). From 2012 to 2018, CysC concentration of each group showed a 
downward trend year by year. In group 1, the mean level of CysC was significantly lower in 2013 than that in 2012 ( $\mathrm{t}=-25.63, \mathrm{P}<0.0001)$. Significant differences in CysC averages were also found between other five years and 2012. Results showed decreased levels of CysC from 2013 to 2018 when compared with 2012 in group 2 to group 7. Generally, these findings reflected that the mean level of CysC was declined year by year in every group. In addition, increased CysC expressions were detected in elderly populations compared with those in young people every year (Table 4). For UA level, in group 1 , it was increased from $340.0 \pm 90.10 \mathrm{umol} / \mathrm{L}$ in 2012 to $344.98 \pm 99.78 \mathrm{umol} / \mathrm{L}$ in 2016 . Similarly, the average levels of UA were gradually increased in years 2012 to 2018 in group 2 to group 4 . There was a significant difference in concentrations of this indicator between 2014 and 2012 in group $2(t=8.87, P<0.0001)$. Elevated UA levels in years 2015 to 2018 were detected as compared to that in 2012. Interestingly, there was a higher mean level of UA in the elderly population when compared to that in young population (Supplementary Table 7). Additionally, Cr expressions in 7 subgroups showed a downtrend year by year. In group 1, it was declined from $76.30 \pm 15.22 \mathrm{umol} / \mathrm{L}$ in 2012 to $65.45 \pm 14.77 \mathrm{umol} / \mathrm{L}$ in 2018 . There was a significant difference for the mean level of $\mathrm{Cr}$ in years 2013 to 2018 compared with 2012 in group 1. Consistent with other indicators, increased concentration of $\mathrm{Cr}$ in older population was observed when compared to that in younger population. For example, the average level of $\mathrm{Cr}$ was $76.30 \pm 15.22 \mathrm{umol} / \mathrm{L}$ in group 1 and $93.03 \pm 21.81 \mathrm{umol} / \mathrm{L}$ in group 7 in 2012 (Supplementary Table 8). Subgroup analysis showed that the UN level in 7 groups declined year by year from 2012 to 2018. When we discussed the level of UN in 2018 and 2012 in group 1, a significant difference was found ( $t=-3.98, P<0.0001)$. Similarly, in other 6 groups, we also observed significant differences in UN concentration between years 2013-2018 and 2012. Results also showed an increased UN level in older groups as compared to that in young people (Supplementary Table 9).

\section{Discussion}

Renal function impairment is often asymptomatic. Many metabolic abnormalities may promote the development of CKD. Preclinical examination has great advantages in preventing CKD from progressing to end-stage renal disease (ESRD) [11]. The present study aimed to describe distribution characteristics of four renal function indicators in the population, including CysC, UA, $\mathrm{Cr}$ and UN. A total of 14,716 health examination population from Chengdu city and its vicinity in Sichuan province from 2012 to 2018 were enrolled in this study. Results revealed that UA levels were increased year by year from 2012 to 2018 both in males and females. However, the other three indicators' levels were declined in years 2012 to 2018 both in two genders. Increased concentrations were found in males compared with those in females every year. When dividing participants into 7 groups by age, we found that UA level was generally increased from 2012 to 2018 in every group. On the contrary, the concentrations of the other three indexes decreased year by year in all subgroups. Notably, the elderly have higher levels of all four kidney indicators than those in the young.

Previous research reported higher levels of serum uric acid (SUA) in males compared with those in females [15]. In 2019, the prevalence of hyperuricemia (HUA) in the whole elderly population was $22.2 \%$, in which it was significantly higher in elderly men than that of elderly women [16]. Similarly, there was a 
correlation between HUA and age [17]. Elevated SUA concentrations in the older population were found by Chang et al [18]. Increased SUA levels in women aged more than 50 were observed. However, the increase disappeared after excluding menopause and other age-related factors, suggesting a strong association between elevated uric acid and age [19]. It is accepted that a higher alcohol consumption in male and the promoting effect of estrogens on UA secretion in females may contribute to this phenomenon. In addition, postmenopausal women presented higher levels of SUA, which could explain increased UA expression in our older females [15]. Clinical studies have demonstrated that decreased serum UA is of great significance in protecting renal function, improving endothelial dysfunction and reducing cardiovascular risk [20]. Emerging researches detected an increased risk of CKD associated with SUA levels [21]. Elevated SUA concentrations have potential to independently predict the development of CKD. In addition, increased UA levels in rats may lead to glomerular hypertension and kidney disease. The process is mediated by arteriolosclerosis, glomerular injury, and tubulointerstitial fibrosis [22]. Collectively, these findings indicated that there was a gender difference in UA levels and it was higher in elderly population than that in young population, which is consistent with our result.

GFR is an important index to evaluate kidney function. It is widely accepted that both $\mathrm{Cr}$ and CysC could be used to estimate GFR. $\mathrm{Cr}$ in the blood is mainly produced as a metabolite of muscle activity and is excreted daily by the kidney with urine. When renal function is impaired, $\mathrm{Cr}$ products cannot be completely excreted, resulting in elevated serum $\mathrm{Cr}$ concentration [23]. Bertille et al. reported increased levels of $\mathrm{Cr}$ and CysC in males when compared with those in females, and they were higher in subjects over 55 years old [24]. Similar to these findings, increased $\mathrm{Cr}$ and CysC levels in males and the elderly population were also detected in our study. In addition, A strong correlation of CKD and high Cr levels was reported [25]. Thus, monitoring the expression of $\mathrm{Cr}$ could effectively prevent the occurrence of CKD.

The level of blood urea nitrogen (BUN) may associate with decreased renal function [26]. Several external factors will influence BUN level, including protein intake, catabolic status, heparin synthesis. Increased UN may indicate renal hypoperfusion due to hypovolemia, nephrovascular disease, or reduced cardiac output [25]. In CKD patients, the decrease of renal function was accompanied by the elevation of BUN. Moreover, higher BUN level may be related to poor renal prognosis in moderately and severely CKD patients [27]. A recent survey showed that BUN was an independent risk factor for CKD in patients with maintenance of renal function [28]. Chang et al. found that elevated UN may accelerate the progression of CKD to dialysis and death [29]. In brief, higher UN expression was not a good signal. In our study, high UN concentrations were detected in men and the elderly, reminding us to monitor the renal function indexes of high-risk groups regularly to prevent or alleviate CKD.

In fact, many previous studies have reported that the prevalence of CKD goes up with age. In view of the high prevalence of CKD in the elderly and the growing elderly population, CKD has become an increasingly serious public health problem [30]. In our study, four renal function indicators showed similar changes with age. According to the global burden of disease study 2013, many risk factors of CKD have been identified, such as diabetes, hypertension, glomerulonephritis [31]. Prevention and control of these related diseases may reduce the risk of CKD. In addition, an observational study lasting over 10 years 
showed that the risk of CKD was significantly increased in non-diabetic individuals who ate foods with higher carbohydrate concentrations, suggesting that dietary adjustment may be beneficial to control the occurrence and development of CKD [32]. Dietary interventions have been used to prevent or slow down the adverse prognosis of CKD in ways that affect kidney and risk factors for CKD. A 23-year follow-up study reported that eating nuts and beans may reduce the risk of kidney disease progression in the population. These two foods are rich in magnesium, which can reduce the secretion of inflammatory and atherogenic cytokines by endothelial cells and protect renal function [33-35]. Studies on the association between alcohol intake and CKD risk have been inconsistent. A meta-analysis reported that healthy adult men with high alcohol intake had a 0.72 -fold lower risk of chronic kidney disease than those who drank less frequently [36].

There are some limitations in our study. First, due to the limitations of cross-section design, the present study failed to analysis the causal relationship between four renal indicators and risk factors. Second, participants were only collected from Chengdu and its vicinity in Sichuan Province, thus, the conclusion could not be extended to the whole country or even to a wider area. Third, our study failed to divide physical examination populations into abnormal and normal group, therefore, the specific distribution of indicators is unclear. However, our research also has some advantages. For instance, our study owns a large sample, and the data were collected through years of follow-up, which made the conclusion more credible and convincing.

\section{Conclusion}

The present study suggested increased levels of CysC, UA, Cr and UN in the elderly population compared with those in the young, and they were higher in males than those in females. This is undoubtedly a reminder to the whole society to monitor early changes in renal indicators to prevent possible adverse outcomes.

\section{Abbreviations}

Cr, creatinine; UA, uric acid; CysC: cystatin C; UN, urea nitrogen; CKD, chronic kidney disease; GFR, glomerular filtration rate.

\section{Declarations}

\section{Acknowledgements}

We thank the participants attended in this study.

Author contributions Rui-Cen Li, Writing-original draft; Ting Bao, Han-Wei Yang, Formal analysis, Investigation and methodology; Wang-Dong Xu, Huai-Rong Tang, funding acquisition, writing-original draft and review-editing 
Funding This work was partly supported by grants from the National Key Research \& Development Program of China (2017YFC0907504), Sichuan Provincial Science and Technology Project (2018SZ0261), and Sichuan Provincial Cadre Health Care Research Project (2017-119).

Conflict of Interest None to declare.

Data Availability Data were available from the corresponding author upon request.

Ethics approval Approval was obtained from Research Committee of West China Hospital, Sichuan University.

\section{Consent for publication}

All of the subjects provided written informed consent for publication.

\section{References}

1. Jha V, Garcia-Garcia G, Iseki K, Li Z, Naicker S, Plattner B, et al. Chronic kidney disease: global dimension and perspectives. Lancet. 2013;382(9888):260-72.

2. Song N, Thaiss F, Guo L. NFkB and Kidney Injury. Front Immunol. 2019;10:815.

3. Sawitzki B. MAIT Cells as Drivers of Renal Fibrosis and CKD. J Am Soc Nephrol. 2019;30(7):1145-46.

4. Ozieh MN, Gebregziabher M, Ward RC, Taber DJ, Egede LE. Creating a 13-year National Longitudinal Cohort of veterans with chronic kidney disease. BMC Nephrol. 2019;20(1):241.

5. Liu ZH, Li G, Zhang L, Chen J, Chen X, Zhao J, et al. Executive Summary: Clinical Practice Guideline of Chronic Kidney Disease - Mineral and Bone Disorder (CKD-MBD) in China. Kidney Dis (Basel). 2019;5(4):197-203.

6. Kumela Goro K, Desalegn Wolide A, Kerga Dibaba F, Gashe Fufa F, Wakjira Garedow A, Edilu Tufa B, et al. Patient Awareness, Prevalence, and Risk Factors of Chronic Kidney Disease among Diabetes Mellitus and Hypertensive Patients at Jimma UniversityMedical Center, Ethiopia. Biomed Res Int. 2019;2019:2383508.

7. Perales-Quintana MM, Saucedo AL, Lucio-Gutiérrez JR, Waksman N, Alarcon-Galvan G, Govea-Torres $\mathrm{G}$, et al. Metabolomic and biochemical characterization of a new model of the transition of acute kidneyinjury to chronic kidney disease induced by folic acid. PeerJ. 2019;7:e7113.

8. Owens EP, Vesey DA, Kassianos AJ, Healy H, Hoy WE, Gobe GC. Biomarkers and the role of mast cells as facilitators of inflammation and fibrosis in chronic kidney disease. Transl Androl Urol. 20198(Suppl 2):S175-83.

9. Ozieh MN, Gebregziabher M, Ward RC, Taber DJ, Egede LE. Creating a 13-year National Longitudinal Cohort of veterans with chronickidney disease. BMC Nephrol. 2019;20(1):241.

10. Musgrove J, Wolf M. Regulation and Effects of FGF23 in Chronic Kidney Disease. Regulation and Effects of FGF23 in Chronic Kidney Disease. Annu Rev Physiol. 2020;82:365-90. 
11. Bellasi A, Di Lullo L, Di lorio B. Chronic Kidney Disease: The Silent Epidemy. J Clin Med. 2019;8(11).

12. Darcy Groesbeck, Anna Köttgen, Rulan Parekh, Elizabeth Selvin, George J. Schwartz, Josef Coresh, et al. Age, Gender, and Race Effects on Cystatin C Levels in US Adolescents. Clin J Am Soc Nephrol. 2008; 3(6): 1777-85.

13. Perales-Quintana MM, Saucedo AL, Lucio-Gutiérrez JR, Waksman N, Alarcon-Galvan G, Govea-Torres $\mathrm{G}$, et al. Metabolomic and biochemical characterization of a new model of the transition of acute kidneyinjury to chronic kidney disease induced by folic acid. PeerJ. 2019;7:e7113.

14. Emadi E, Abdoli N, Ghanbarinejad V, Mohammadi HR, Mousavi Mobarakeh K, Azarpira N, et al. The potential role of mitochondrial impairment in the pathogenesis of imatinib-induced renal injury. Heliyon. 2019;5(6):e01996.

15. Kamei K, Konta T, Hirayama A, Suzuki K, Ichikawa K, Fujimoto S, et al. A slight increase within the normal range of serum uric acid and the decline in renal function: associations in a communitybased population. Nephrol Dial Transplant. 2014;29(12):2286-92.

16. Yang Y, Zhou W, Wang Y, Zhou R. Gender-specific association between uric acid level and chronic kidney disease in the elderlyhealth checkup population in China. Ren Fail. 2019;41(1):197-203.

17. Yang WX, Ma Y, Hou YL, Wang YB, You CG. Prevalence of Hyperuricemia and its Correlation with Serum Lipids and Blood Glucose in Physical Examination Population in 2015 - 2018: a Retrospective Study. Clin Lab. 2019;65(8).

18. Chang HY, Lee PH, Lei CC, Tung CW, Hsu YC, Huang TJ, et al. Hyperuricemia is an independent risk factor for new onset micro-albuminuria in a middle-aged and elderly population: a prospective cohort study in taiwan. PLoS One. 2013;8(4):e61450.

19. Hak AE, Choi HK. Menopause, postmenopausal hormone use and serum uric acid levels in US women-the Third National Health and Nutrition Examination Survey. Arthritis Res Ther. 2008;10(5):R116.

20. Kasahara M, Kuwabara Y, Moriyama T, Tanabe K, Satoh-Asahara N, Katsuya T, et al. Intensive uric acid-lowering therapy in CKD patients: the protocol for a randomized controlled trial. Clin Exp Nephrol. 2020;24(3):235-41.

21. Mallat SG, Al Kattar S, Tanios BY, Jurjus A. Hyperuricemia, Hypertension, and Chronic Kidney Disease: an Emerging Association. Curr Hypertens Rep. 2016;18(10):74.

22. Johnson RJ, Nakagawa T, Jalal D, Sánchez-Lozada LG, Kang DH, Ritz E. Uric acid and chronic kidney disease: which is chasing which? Nephrol Dial Transplant. 2013;28(9):2221-8.

23. Chang HL, Wu CC, Lee SP, Chen YK, Su W, Su SL. A predictive model for progression of CKD. Medicine (Baltimore). 2019;98(26):e16186.

24. Edinga-Melenge BE, Yakam AT, Nansseu JR, Bilong C, Belinga S, Minkala E, et al Reference intervals for serum cystatin $\mathrm{C}$ and serum creatinine in an adult sub-Saharan Africanpopulation. BMC Clin Pathol. 2019;19:4.

25. Chala G, Sisay T, Teshome Y. Chronic Kidney Disease And Associated Risk Factors Among Cardiovascular Patients. Int J Nephrol Renovasc Dis. 2019;12:205-11. 
26. Dossetor JB. Creatininemia versus uremia. The relative significance of blood urea nitrogen and serumcreatinine concentrations in azotemia. Ann Intern Med. 1966;65(6):1287-99.

27. Seki M, Nakayama M, Sakoh T, Yoshitomi R, Fukui A, Katafuchi E, et al. Blood urea nitrogen is independently associated with renal outcomes in Japanese patients with stage 3-5 chronic kidney disease: a prospectiveobservational study. BMC Nephrol. 2019;20(1):115.

28. Kuwabara M, Hisatome I, Roncal-Jimenez CA, Niwa K, Andres-Hernando A, Jensen T, et al. Increased Serum Sodium and Serum Osmolarity Are Independent Risk Factors for Developing Chronic Kidney Disease; 5 Year Cohort Study. PLoS One. 2017;12(1):e0169137.

29. Desai AS, Toto R, Jarolim P, Uno H, Eckardt KU, Kewalramani R, et al. Association between cardiac biomarkers and the development of ESRD in patients with type 2 diabetes mellitus, anemia, and CKD. Am J Kidney Dis. 2011;58(5):717-28.

30. Zonoozi S, Ramsay SE, Papacosta O, Lennon LT, Ellins EA, Halcox JPJ, et al. Chronic kidney disease, cardiovascular risk markers and total mortality in older men: cystatin $\mathrm{C}$ versus creatinine.SG. J Epidemiol Community Health. 2019;73(7):645-51.

31. GBD 2013 Mortality and Causes of Death Collaborators. Global, regional, and national age-sex specific all-cause and cause-specificmortality for 240 causes of death, 1990-2013: a systematic analysis for the Global Burden of Disease Study 2013. Lancet. 2015;385(9963):117-71.

32. Nam KH, An SY, Joo YS, Lee S, Yun HR, Jhee JH, et al. Carbohydrate-Rich Diet Is Associated with Increased Risk of Incident ChronicKidney Disease in Non-Diabetic Subjects. J Clin Med. 2019;8(6).

33. Rebholz CM, Crews DC, Grams ME, Steffen LM, Levey AS, Miller ER 3rd, et al. DASH (Dietary Approaches to Stop Hypertension) Diet and Risk of Subsequent Kidney Disease. Am J Kidney Dis. 2016;68(6):853-61.

34. Ferrè S, Baldoli E, Leidi M, Maier JA. Magnesium deficiency promotes a pro-atherogenic phenotype in cultured human endothelial cells via activation of NFkB. Biochim Biophys Acta. 2010;1802(11):9528.

35. Tin A, Grams ME, Maruthur NM, Astor BC, Couper D, Mosley TH, et al. Results from the Atherosclerosis Risk in Communities study suggest that low serum magnesium is associated with incident kidney disease. Kidney Int. 2015;87(4):820-7.

36. Cheungpasitporn W, Thongprayoon C, Kittanamongkolchai W, Brabec BA, O'Corragain OA, Edmonds PJ, et al. High alcohol consumption and the risk of renal damage: a systematic review and metaanalysis. QJM. 2015;108(7):539-48.

\section{Tables}

Table 1 Baseline characteristics of participants in this study from 2012 to 2018 ( $n=14716)$ 


\begin{tabular}{lc}
\hline Characteristics & Outcomes \\
\hline $\begin{array}{c}\text { Sex, } \mathrm{n}(\%) \\
\text { Male }\end{array}$ & $8148(55.37 \%)$ \\
\hline Female & $6568(44.63 \%)$ \\
\hline Age, year & $45.12 \pm 13.13$ \\
\hline Age group, $\mathrm{n}(\%)$ & \\
\hline $20 \sim$ & $1608(10.93 \%)$ \\
\hline $30 \sim$ & $3683(25.03 \%)$ \\
\hline $40 \sim$ & $5005(34.01 \%)$ \\
\hline $50 \sim$ & $2378(16.16)$ \\
\hline $60 \sim$ & $1084(7.37 \%)$ \\
\hline $70 \sim$ & $819(5.57 \%)$ \\
$80 \sim$ & $139(0.94 \%)$ \\
\hline BMI, kg/m & $23.36 \pm 3.14$ \\
\hline Waist hip ratio & $0.85 \pm 0.10$ \\
\hline SBP, $\mathrm{mmHg}$ & $113.30 \pm 15.34$ \\
\hline DBP, $\mathrm{mmHg}$ & $73.52 \pm 9.68$ \\
\hline
\end{tabular}

BMI, body mass index; SBP, systolic blood pressure; DBP, diastolic blood pressure

Table 2 Characteristics of cystatin C among all the health examination population (2012-2018)

\begin{tabular}{ccccc}
\hline Year & Mean $\pm S$ & Min, Max & Statistics & $P$ value \\
\hline 2012 & $0.92 \pm 0.20$ & $0.09,9.20$ & - & $-\overline{-}$ \\
2013 & $0.87 \pm 0.17$ & $0.11,9.61$ & $\mathrm{t}=-55.82$ & $<0.0001$ \\
\hline 2014 & $0.87 \pm 0.17$ & $0.05,9.15$ & $\mathrm{t}=-54.66$ & $<0.0001$ \\
\hline 2015 & $0.87 \pm 0.18$ & $0.06,11.06$ & $\mathrm{t}=-42.87$ & $<0.0001$ \\
\hline 2016 & $0.87 \pm 0.17$ & $0.38,7.75$ & $\mathrm{t}=-54.00$ & $<0.0001$ \\
\hline 2017 & $0.83 \pm 0.17$ & $0.36,9.03$ & $\mathrm{t}=-99.27$ & $<0.0001$ \\
\hline 2018 & $0.83 \pm 0.18$ & $0.03,8.08$ & $\mathrm{t}=-90.57$ & $<0.0001$ \\
\hline
\end{tabular}

Table 3 Characteristics of all the health examination population with cystatin C in different sex

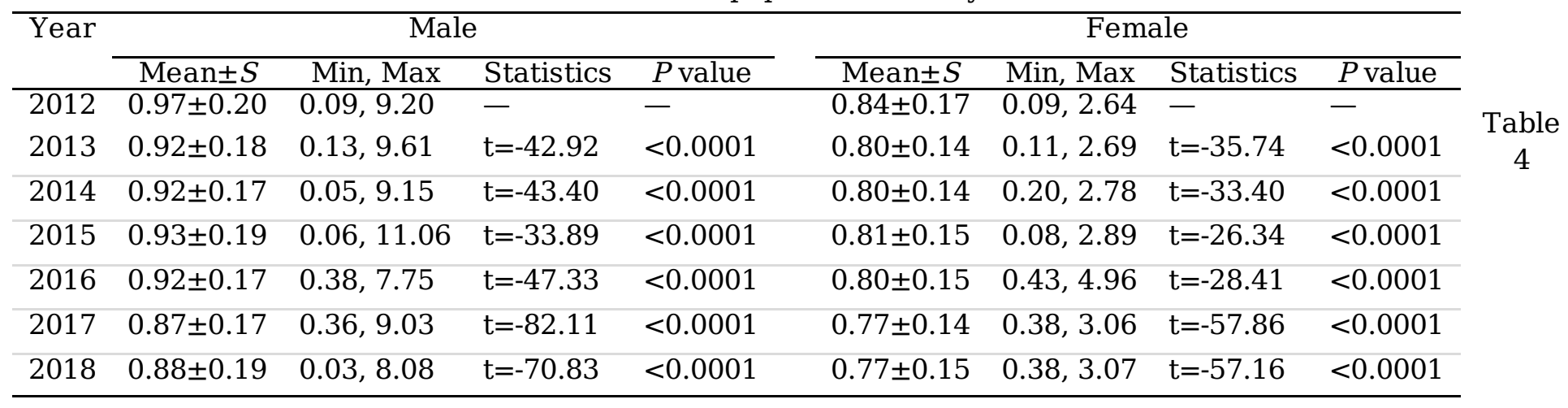

Characteristics of all the health examination population with cystatin $\mathrm{C}$ in different ages

\section{Supplementary Files}




\begin{tabular}{|c|c|c|c|c|c|}
\hline Age group & Year & Mean $\pm S$ & Min, Max & Statistics & $P$ value \\
\hline \multirow[t]{7}{*}{$20 \sim$} & 2012 & $0.86 \pm 0.12$ & $0.33,1.49$ & - & - \\
\hline & 2013 & $0.80 \pm 0.11$ & $0.40,1.16$ & $t=-25.63$ & $<0.0001$ \\
\hline & 2014 & $0.80 \pm 0.11$ & $0.36,1.22$ & $\mathrm{t}=-21.63$ & $<0.0001$ \\
\hline & 2015 & $0.80 \pm 0.11$ & $0.46,1.27$ & $\mathrm{t}=-19.45$ & $<0.0001$ \\
\hline & 2016 & $0.79 \pm 0.09$ & $0.51,1.16$ & $\mathrm{t}=-24.54$ & $<0.0001$ \\
\hline & 2017 & $0.74 \pm 0.10$ & $0.42,1.14$ & $t=-42.16$ & $<0.0001$ \\
\hline & 2018 & $0.74 \pm 0.09$ & $0.50,1.00$ & $\mathrm{t}=-42.72$ & $<0.0001$ \\
\hline \multirow[t]{7}{*}{$30 \sim$} & 2012 & $0.85 \pm 0.13$ & $0.34,1.47$ & - & - \\
\hline & 2013 & $0.81 \pm 0.11$ & $0.15,1.46$ & $\mathrm{t}=-28.41$ & $<0.0001$ \\
\hline & 2014 & $0.81 \pm 0.11$ & $0.07,1.44$ & $\mathrm{t}=-25.90$ & $<0.0001$ \\
\hline & 2015 & $0.82 \pm 0.12$ & $0.43,2.13$ & $t=-23.45$ & $<0.0001$ \\
\hline & 2016 & $0.80 \pm 0.10$ & $0.38,1.69$ & $\mathrm{t}=-32.34$ & $<0.0001$ \\
\hline & 2017 & $0.76 \pm 0.10$ & $0.45,1.41$ & $t=-59.78$ & $<0.0001$ \\
\hline & 2018 & $0.76 \pm 0.10$ & $0.38,1.71$ & $t=-58.27$ & $<0.0001$ \\
\hline \multirow[t]{7}{*}{$40 \sim$} & 2012 & $0.88 \pm 0.19$ & $0.13,9.20$ & - & - \\
\hline & 2013 & $0.83 \pm 0.13$ & $0.11,2.47$ & $t=-25.66$ & $<0.0001$ \\
\hline & 2014 & $0.83 \pm 0.12$ & $0.18,2.12$ & $t=-26.19$ & $<0.0001$ \\
\hline & 2015 & $0.83 \pm 0.13$ & $0.36,2.50$ & $\mathrm{t}=-18.34$ & $<0.0001$ \\
\hline & 2016 & $0.81 \pm 0.11$ & $0.39,2.01$ & $\mathrm{t}=-24.56$ & $<0.0001$ \\
\hline & 2017 & $0.77 \pm 0.11$ & $0.36,1.48$ & $t=-50.67$ & $<0.0001$ \\
\hline & 2018 & $0.77 \pm 0.11$ & $0.03,1.68$ & $t=-50.57$ & $<0.0001$ \\
\hline \multirow[t]{7}{*}{$50 \sim$} & 2012 & $0.96 \pm 0.17$ & $0.12,4.75$ & - & - \\
\hline & 2013 & $0.90 \pm 0.22$ & $0.41,9.61$ & $t=-19.62$ & $<0.0001$ \\
\hline & 2014 & $0.89 \pm 0.20$ & $0.07,9.15$ & $t=-20.28$ & $<0.0001$ \\
\hline & 2015 & $0.90 \pm 0.22$ & $0.08,11.06$ & $t=-15.24$ & $<0.0001$ \\
\hline & 2016 & $0.88 \pm 0.18$ & $0.46,7.75$ & $t=-20.58$ & $<0.0001$ \\
\hline & 2017 & $0.84 \pm 0.19$ & $0.39,9.03$ & $t=-36.35$ & $<0.0001$ \\
\hline & 2018 & $0.83 \pm 0.17$ & $0.40,8.08$ & $\mathrm{t}=-34.02$ & $<0.0001$ \\
\hline \multirow[t]{7}{*}{$60 \sim$} & 2012 & $1.06 \pm 0.18$ & $0.09,1.74$ & - & - \\
\hline & 2013 & $0.98 \pm 0.14$ & $0.62,1.84$ & $t=-18.93$ & $<0.0001$ \\
\hline & 2014 & $0.95 \pm 0.14$ & $0.05,1.94$ & $\mathrm{t}=-20.80$ & $<0.0001$ \\
\hline & 2015 & $0.96 \pm 0.16$ & $0.06,2.82$ & $t=-15.13$ & $<0.0001$ \\
\hline & 2016 & $0.94 \pm 0.13$ & $0.60,1.97$ & $\mathrm{t}=-14.49$ & $<0.0001$ \\
\hline & 2017 & $0.89 \pm 0.13$ & $0.58,1.77$ & $t=-30.17$ & $<0.0001$ \\
\hline & 2018 & $0.89 \pm 0.15$ & $0.43,3.07$ & $t=-25.31$ & $<0.0001$ \\
\hline \multirow[t]{7}{*}{$70 \sim$} & 2012 & $1.10 \pm 0.20$ & $0.63,2.10$ & - & - \\
\hline & 2013 & $1.08 \pm 0.20$ & $0.60,2.13$ & $\mathrm{t}=-21.32$ & $<0.0001$ \\
\hline & 2014 & $1.08 \pm 0.20$ & $0.58,2.27$ & $\mathrm{t}=-16.74$ & $<0.0001$ \\
\hline & 2015 & $1.06 \pm 0.22$ & $0.62,4.96$ & $\mathrm{t}=-19.28$ & $<0.0001$ \\
\hline & 2016 & $1.01 \pm 0.18$ & $0.63,2.76$ & $t=-25.74$ & $<0.0001$ \\
\hline & 2017 & $1.00 \pm 0.20$ & $0.58,2.75$ & $t=-19.09$ & $<0.0001$ \\
\hline & 2018 & $1.10 \pm 0.20$ & $0.63,2.10$ & $\mathrm{t}=-22.27$ & $<0.0001$ \\
\hline \multirow[t]{7}{*}{$80 \sim$} & 2012 & $1.35 \pm 0.32$ & $0.78,2.64$ & - & - \\
\hline & 2013 & $1.22 \pm 0.23$ & $0.80,2.48$ & $t=-8.19$ & $<0.0001$ \\
\hline & 2014 & $1.21 \pm 0.26$ & $0.72,2.78$ & $\mathrm{t}=-7.98$ & $<0.0001$ \\
\hline & 2015 & $1.22 \pm 0.29$ & $0.15,2.89$ & $\mathrm{t}=-4.77$ & $<0.0001$ \\
\hline & 2016 & $1.20 \pm 0.26$ & $0.70,2.57$ & $\mathrm{t}=-4.78$ & $<0.0001$ \\
\hline & 2017 & $1.15 \pm 0.26$ & $0.66,2.45$ & $\mathrm{t}=-7.28$ & $<0.0001$ \\
\hline & 2018 & $1.17 \pm 0.32$ & $0.63,4.65$ & $\mathrm{t}=-2.22$ & 0.0286 \\
\hline
\end{tabular}

